Portland State University

PDXScholar

8-1-2009

\title{
A Discrete Impulsive Model for Random Heating and Brownian Motion
}

John D. Ramshaw

Portland State University, jdramshaw@yahoo.com

Follow this and additional works at: https://pdxscholar.library.pdx.edu/phy_fac

Part of the Physics Commons

Let us know how access to this document benefits you.

Citation Details

Ramshaw, J. D. (2010). A discrete impulsive model for random heating and Brownian motion. American Journal Of Physics, 78(1), 9-13.

This Article is brought to you for free and open access. It has been accepted for inclusion in Physics Faculty Publications and Presentations by an authorized administrator of PDXScholar. Please contact us if we can make this document more accessible: pdxscholar@pdx.edu. 


\title{
A discrete impulsive model for random heating and Brownian motion
}

\author{
John D. Ramshaw \\ Department of Physics, Portland State University, Portland, Oregon 97207
}

(Received 14 May 2009; accepted 10 August 2009)

\begin{abstract}
The energy of a mechanical system subjected to a random force with zero mean increases irreversibly and diverges with time in the absence of friction or dissipation. This random heating effect is usually encountered in phenomenological theories formulated in terms of stochastic differential equations, the epitome of which is the Langevin equation of Brownian motion. We discuss a simple discrete impulsive model that captures the essence of random heating and Brownian motion. The model may be regarded as a discrete analog of the Langevin equation, although it is developed $a b$ initio. Its analysis requires only simple algebraic manipulations and elementary averaging concepts, but no stochastic differential equations (or even calculus). The irreversibility in the model is shown to be a consequence of a natural causal stochastic condition that is closely analogous to Boltzmann's molecular chaos hypothesis in the kinetic theory of gases. The model provides a simple introduction to several ostensibly more advanced topics, including random heating, molecular chaos, irreversibility, Brownian motion, the Langevin equation, and fluctuation-dissipation theorems. (C) 2010 American Association of Physics Teachers.
\end{abstract}

[DOI: $10.1119 / 1.3213526]$

\section{INTRODUCTION}

The idealization of an isolated physical system is just that - an idealization. All real physical systems interact with their surroundings. Those interactions are sometimes negligible, but are frequently of essential importance. Their details are often intractably complicated, and are commonly modeled as random perturbations. The classic example of this approach is the Langevin theory of Brownian motion, ${ }^{1-3}$ in which the equation of motion of a large particle immersed in a bath of small particles is taken to be

$$
m \frac{d \mathbf{v}}{d t}=-\beta \mathbf{v}+\mathbf{F}(t),
$$

where $m$ is the mass of the particle, $\mathbf{v}$ is its velocity, $\beta>0$ is a friction coefficient, and $\mathbf{F}(t)$ is a random force which rapidly fluctuates about a mean value of zero.

The Langevin approach has been very successful and has been fruitfully generalized in many directions. In doing so, it has not always been recognized that the friction term is essential to counterbalance the heating produced by the random force, thereby enabling the system to approach and attain thermal equilibrium. ${ }^{4-6}$ In the absence of friction (that is, when $\beta=0$ ), the mean kinetic energy of the particle diverges with time due to the irreversible heating produced by the random force, but this behavior is not immediately obvious upon casual inspection of Eq. (1).

The fact that a random force with zero mean produces irreversible heating may seem surprising at first-if the random force averages to zero, then why does the random heating it produces not likewise average to zero? And where does the irreversibility come from? The original motivation for this paper was to provide a simple physical explanation for this underappreciated and perhaps counterintuitive effect in terms of a simple impulsive model. The explanation turned out to be mathematically simple but conceptually subtle, and it led to some unexpected insights that are less easily extracted from more advanced treatments. Those insights may be of wider interest, and will be discussed in detail.
The frictionless impulsive model that exhibits and explains random heating is easily generalized to include friction. It then becomes a discrete impulsive analog of Eq. (1), and thereby provides a simple introduction to the ideas of the Langevin theory of Brownian motion, as well as the essence of fluctuation-dissipation theorems. However, the model will be motivated and formulated independently of Eq. (1), and thus requires no previous exposure to Brownian motion or the Langevin equation.

The model has the pedagogical advantage that it requires only simple algebraic manipulations, thereby minimizing the danger of the mathematics obscuring the physics. It should therefore be easily accessible to undergraduate physics and engineering students. The model requires an intuitive grasp of some basic probabilistic concepts, namely statistical averaging and correlations between random variables. Readers lacking this background will find a brief summary of the essentials in the Appendix.

\section{IMPULSIVE MODEL FOR RANDOM HEATING}

Consider a particle that is constrained to move along a horizontal line with no friction. The particle experiences a sequence of random impulsive forces, or equivalently accelerations. Each impulse produces a random discontinuous jump in the velocity $v$ of the particle. Between impulses, $v$ remains constant. The random heating effect will manifest itself as an irreversible increase in the particle's kinetic energy per unit mass $v^{2} / 2$. The value of $v$ after the $n$th impulse but before the $(n+1)$ st impulse will be denoted by $v_{n}$. The initial velocity of the particle prior to the first impulse is therefore $v_{0}$. It is understood that the index $n$ increases with time, and vice versa. The time intervals between the impulses need not be constant, but they do not explicitly enter into the model and hence need not be specified.

The velocity jump produced by the $n$th impulse will be denoted by $\Delta v_{n}$. The velocity of the particle therefore satisfies the discrete evolution equation 


$$
v_{n}=v_{n-1}+\Delta v_{n}
$$

The velocity increments $\Delta v_{n}$ are assumed to be statistically independent random variables with zero mean values. Statistical averages will be denoted by angular brackets, so that $\langle V\rangle$ is the statistical average of the random variable $V$. The mean value of $\Delta v_{n}$ is $\left\langle\Delta v_{n}\right\rangle=0$, and its mean-squared value is $\left\langle\Delta v_{n}^{2}\right\rangle$. We further assume that the random increments $\Delta v_{n}$ are all obtained by sampling from the same probability distribution, so that $\left\langle\Delta v_{n}^{2}\right\rangle$ has the same value $\left\langle\Delta v^{2}\right\rangle$ for all $n$.

Equation (2) is the fundamental equation of the model. It could hardly be any simpler mathematically, and yet we shall see that it contains a wealth of interesting physics. We now proceed to analyze how the irreversible random heating follows from Eq. (2). Two complementary derivations will be given, and a consideration of how they are related will provide considerable insight into the origin of the irreversibility.

The first derivation proceeds by squaring Eq. (2) to obtain

$$
v_{n}^{2}=v_{n-1}^{2}+2 v_{n-1} \Delta v_{n}+\Delta v_{n}^{2}
$$

followed by taking the statistical average of Eq. (3). Averaging is a linear operation, so we obtain

$$
\left\langle v_{n}^{2}\right\rangle=\left\langle v_{n-1}^{2}\right\rangle+2\left\langle v_{n-1} \Delta v_{n}\right\rangle+\left\langle\Delta v^{2}\right\rangle .
$$

Now focus attention on the quantity $C_{n} \equiv\left\langle v_{n-1} \Delta v_{n}\right\rangle$, which is the statistical correlation between $v_{n-1}$ and $\Delta v_{n}$. If those quantities were statistically independent, then $C_{n}$ would factor into the product of their averages and would have the value $C_{n}=\left\langle v_{n-1}\right\rangle\left\langle\Delta v_{n}\right\rangle=0$. But $v_{n-1}$ and $\Delta v_{n}$ are indeed statistically independent, because $v_{n-1}$ is the value of $v$ prior to the arrival of $\Delta v_{n}$, and therefore has not yet been influenced by $\Delta v_{n}$. Moreover, there can be no indirect correlation between $v_{n-1}$ and $\Delta v_{n}$ via the previous impulses that have influenced $v_{n-1}$ because $\Delta v_{n}$ is statistically independent of all those previous impulses. That is to say, $\Delta v_{n}$ has no memory and no knowledge of anything that preceded it, including the value of $v$ that the particle possessed just prior to its occurrence. In essence, this property is a matter of causality; the impulses $\Delta v_{n}$ are the causes and the velocities $v_{n}$ are their effects, and the latter cannot precede the former. We therefore conclude that $C_{n}=0$, so that $\mathrm{Eq}$. (4) reduces to

$$
\left\langle v_{n}^{2}\right\rangle=\left\langle v_{n-1}^{2}\right\rangle+\left\langle\Delta v^{2}\right\rangle .
$$

Because $\left\langle\Delta v^{2}\right\rangle>0$, Eq. (5) implies that the energy of the particle irreversibly increases with time. This increase is the phenomenon of random heating, which is therefore seen to be a straightforward consequence of the simple impulsive model of Eq. (2). The relation $C_{n}=0$ is closely analogous to Boltzmann's famous Stosszahlansatz or molecular chaos hypothesis in the kinetic theory of gases, ${ }^{1,7}$ according to which a pair of colliding molecules is assumed to be statistically uncorrelated just before the collision (but not afterward). That hypothesis is now known to be the origin of the irreversibility in Boltzmann's equation, which was not obvious when that equation was first formulated.

In contrast to $C_{n}$, the correlation $\left\langle v_{n} \Delta v_{n}\right\rangle$ is nonzero due to the fact that $v_{n}$ has just been influenced by $\Delta v_{n}$ via Eq. (2). The value of $\left\langle v_{n} \Delta v_{n}\right\rangle$ can be determined by solving Eq. (2) for $v_{n-1}$, squaring, averaging, and comparing the result with Eq. (5). We thereby obtain

$$
\left\langle v_{n} \Delta v_{n}\right\rangle=\left\langle\Delta v^{2}\right\rangle
$$

which is positive as expected, because the particle is more likely to be found moving in the direction it was last pushed than in the opposite direction. The difference between $C_{n}$ and $\left\langle v_{n} \Delta v_{n}\right\rangle$ reflects the asymmetry or distinction between the forward and backward directions of time (that is, increasing and decreasing $n$, respectively), which is the essence of irreversibility.

The correlation $\left\langle v_{n} \Delta v_{n}\right\rangle$ is closely related to the average work per unit mass done on the particle by $\Delta v_{n}$, which one would intuitively expect to be given by $\left\langle W_{n}\right\rangle$, where

$$
W_{n} \equiv \frac{1}{2}\left(v_{n}+v_{n-1}\right) \Delta v_{n}
$$

One might think that the average velocity at the time of the impulse should be weighted by the time intervals between impulses, but that procedure would not be sensible in the present context because the time intervals are arbitrary and do not enter into the model in any way. Thus there is no basis for weighting the particle velocities before and after the impulse unequally, so that the use of the simple arithmetical average velocity in Eq. (7) is required by symmetry. Because $C_{n}=0$, Eq. (7) implies that

$$
\left\langle W_{n}\right\rangle=\frac{1}{2}\left\langle\left(v_{n}+v_{n-1}\right) \Delta v_{n}\right\rangle=\frac{1}{2}\left\langle v_{n} \Delta v_{n}\right\rangle
$$

It follows from Eqs. (5), (6), and (8) that

$$
\left\langle v_{n}^{2}\right\rangle-\left\langle v_{n-1}^{2}\right\rangle=2\left\langle W_{n}\right\rangle
$$

so that $\left\langle W_{n}\right\rangle$ is the average change in the kinetic energy per unit mass of the particle produced by $\Delta v_{n}$, and is therefore the mean work per unit mass done on the particle. Similar questions concerning the correlation between the random force and the particle velocity, and the average work done by the random force on the particle, also arise in the continuous Langevin theory of Eq. (1), where they are somewhat more subtle. These issues have been discussed and resolved by London, ${ }^{8}$ who obtained results analogous to and consistent with those derived more easily above.

In many situations of interest the random impulses are very small but very frequent, and the condition $\left|\Delta v_{n}\right|$ $\ll\left|v_{n-1}\right|$ is usually satisfied. However, if the last term in Eq. (3) had been neglected on that basis, the random heating effect would have been lost. Random heating is inherently quadratic in the random impulses due to the fact that the kinetic energy of the particle is quadratic in $v$, which is why it does not average to zero as the impulses do.

The second derivation is the discrete analog of the usual procedure for analyzing the differential Langevin equation (1). ${ }^{1-3}$ It proceeds by formally solving Eq. (2) for $v_{n}$ in terms of its initial value $v_{0}$. To that end, we set $n=i$ in Eq. (2) and sum from $i=1$ to $i=n$. The intermediate values of $v_{i}$ cancel in pairs, and we obtain

$$
v_{n}=v_{0}+\sum_{i=1}^{n} \Delta v_{i} .
$$

Squaring both sides of Eq. (10) and averaging the result, we find 


$$
\left\langle v_{n}^{2}\right\rangle=v_{0}^{2}+\sum_{i=1}^{n} \sum_{j=1}^{n}\left\langle\Delta v_{i} \Delta v_{j}\right\rangle,
$$

where use has been made of the fact that $\left\langle\Delta v_{i}\right\rangle=0$. The random increments $\Delta v_{n}$ are statistically independent, so they are uncorrelated with each other; that is,

$$
\left\langle\Delta v_{i} \Delta v_{j}\right\rangle=\delta_{i j}\left\langle\Delta v_{i}^{2}\right\rangle+\left(1-\delta_{i j}\right)\left\langle\Delta v_{i}\right\rangle\left\langle\Delta v_{j}\right\rangle=\delta_{i j}\left\langle\Delta v_{i}^{2}\right\rangle,
$$

where $\delta_{i j}$ is the Kronecker delta. Combining Eqs. (11) and (12), we obtain

$$
\left\langle v_{n}^{2}\right\rangle=v_{0}^{2}+\sum_{i=1}^{n}\left\langle\Delta v_{i}^{2}\right\rangle=v_{0}^{2}+n\left\langle\Delta v^{2}\right\rangle,
$$

from which Eq. (5) follows directly without the use of Eq. (4), and hence without the need to explicitly consider $C_{n}$. Although it has not been used, Eq. (4) remains valid, and a comparison of Eqs. (4) and (5) confirms that $C_{n}=0$, for otherwise those two equations would not be consistent.

Both derivations therefore lead to the same result, Eq. (5), and as we have seen both contain the stochastic causality condition $C_{n}=0$ either explicitly or implicitly. This condition is not readily apparent in the second derivation, which was designed to circumvent the need to explicitly consider $C_{n}$. The analogous maneuver is advantageous in connection with Eq. (1) because it avoids the need to deal with the correlation $\langle\mathbf{v}(t) \cdot \mathbf{F}(t)\rangle$, which is more difficult to evaluate and has been a source of considerable confusion. ${ }^{8}$ In the present discrete context, circumventing $C_{n}$ has a corresponding disadvantage: it conceals the stochastic causality condition that implicitly underlies the formulation and gives rise to its irreversibility, whereas the first derivation brings that condition to the fore so that its significance can be appreciated.

The second derivation is so successful at concealing the stochastic causality condition or hypothesis that it is not obvious where and how the latter enters in. The conundrum is that the only statistical information used in the derivation is the correlation in Eq. (12), which is symmetrical in $i$ and $j$ and contains no information from which the forward and backward directions of time can be inferred or distinguished. The manner in which that distinction infiltrates the derivation is subtle, and consists in the fact that causality is implicit in our decision to solve Eq. (2) going forward in time (that is, for $n>0$ ) starting from the initial condition $v_{0}$, rather than backward in time (for $n<0$ ). That decision prevents $v_{n}$ from being influenced by impulses that have not yet occurred; that is, values of $\Delta v_{i}$ for $i>n$. We leave it as an exercise for the reader to verify that if we had instead solved Eq. (2) going backward in time (that is, for $n<0$ ) starting from $v_{0}$, we would have obtained $\left\langle v_{n}^{2}\right\rangle=v_{0}^{2}-n\left\langle\Delta v^{2}\right\rangle$ instead of Eq. (13), so that $\left\langle v_{n}^{2}\right\rangle$ would decrease as $n$ increases, thereby resulting in random cooling rather than heating. Irreversibility enters into the usual treatments of the Langevin equation ${ }^{1-3}$ in exactly the same way. Thus the Langevin formalism for Brownian motion is implicitly based on a causal stochastic condition closely analogous to Boltzmann's molecular chaos hypothesis, and the irreversibility in both the Langevin and Boltzmann equations has basically the same origin. Those equations are the two great pillars or paradigms of nonequilibrium statistical physics, so it is very satisfying to recognize this connection between them.
Finally, we revisit the question raised in Sec. I: the random impulses $\Delta v_{n}$ average to zero, so why does the random heating they produce not also average to zero? The preceding analysis provides an answer to this question in terms of elementary algebra and statistics, but the essence of the situation can be understood more easily by considering the special case in which the random impulses $\Delta v_{n}$ all have the same magnitude $\Delta v>0$, and therefore have only two possible and equally probable values $\pm \Delta v$, which produce equal and opposite changes in the velocity $v$. Equation (3) shows that $v^{2}$ increases more when $v_{n-1} \Delta v>0$ than it decreases when $v_{n-1} \Delta v<0$. Those two cases are equally likely, but the particle gains more energy in the former than it loses back in the latter, so that its energy steadily increases on the average.

This special case is equivalent to an unbiased onedimensional random walk in velocity space. As is well known, the mean-square displacement in a random walk increases linearly with the number of steps, ${ }^{2}$ which is the behavior shown by Eq. (13). Moreover, the mean-square displacement in velocity space is just twice the mean kinetic energy per unit mass of the particle relative to its initial value. This correspondence provides an alternative simple explanation of random heating for students who are already familiar with random walks. ${ }^{2,9-12}$

\section{IMPULSIVE MODEL FOR BROWNIAN MOTION}

Armed with the insights obtained from the frictionless model of Eq. (2), we now proceed to generalize the model to the case in which the particle experiences a deterministic frictional force in addition to random impulsive forces, just as it does in the continuous Langevin equation (1). This generalization will constitute a discrete analog of the full Langevin equation (1), although we shall not explore all of its consequences here. Our attention will be restricted to the mean kinetic energy of the particle and how it differs from the case of zero friction.

Friction may be introduced into Eq. (2) by the addition of a frictional impulse of the form $-\alpha v_{n-1}$, where $\alpha>0$ is a friction coefficient. Equation (2) then becomes

$$
v_{n}=v_{n-1}-\alpha v_{n-1}+\Delta v_{n}=(1-\alpha) v_{n-1}+\Delta v_{n} .
$$

We must require that $\alpha<1$ to ensure that the friction cannot reverse the sign of $v$. However, Eq. (14) shows that $\alpha$ $\sim 1 / N$, where $N$ is the number of impulses or steps required for the accumulated frictional change in $v$ to be of the same order as $v$ itself. We shall assume that $N \gg 1$, so that $\alpha$ satisfies the more restrictive condition $\alpha \ll 1$. Squaring both sides of Eq. (14), averaging the result, and again invoking the stochastic causality condition $C_{n}=0$, we obtain

$$
\left\langle v_{n}^{2}\right\rangle=(1-\alpha)^{2}\left\langle v_{n-1}^{2}\right\rangle+\left\langle\Delta v^{2}\right\rangle .
$$

Because $(1-\alpha)^{2}<1$, Eq. (15) no longer implies an irreversible increase in the energy, but shows that as $n \rightarrow \infty$, the particle approaches a statistically steady state in which $\left\langle v_{n}^{2}\right\rangle$ and $\left\langle v_{n-1}^{2}\right\rangle$ asymptotically approach the common value $\left\langle v^{2}\right\rangle$ determined by 


$$
\left\langle v^{2}\right\rangle=(1-\alpha)^{2}\left\langle v^{2}\right\rangle+\left\langle\Delta v^{2}\right\rangle
$$

Because $\alpha \ll 1$, Eq. (16) may be simplified to

$$
2 \alpha\left\langle v^{2}\right\rangle=\left\langle\Delta v^{2}\right\rangle
$$

Equation (17) expresses the asymptotic value of $\left\langle v^{2}\right\rangle$, and hence the mean kinetic energy per unit mass of the particle, in terms of the mean-squared magnitude of the random impulses and the friction coefficient $\alpha$.

The statistical balance between the random and frictional impulses therefore results in a nonzero asymptotic steadystate value for the mean kinetic energy of the particle. The friction dissipates the energy produced by the random heating and thereby prevents the divergence of kinetic energy that would otherwise occur. This effect is hardly surprising and is easily understood. However, the deceptively simple Eq. (17) is much more significant than it appears-it is prototypical of a broad class of analogous relations called fluctuation-dissipation theorems, ${ }^{1-6}$ which are of central importance in nonequilibrium statistical physics. Like Eq. (17), such relations typically relate statistical fluctuations (in this case the strength of the random impulses) to the dissipation of energy by irreversible processes (in this case the friction coefficient $\alpha$ ), or alternatively to the related response of the system to external perturbations (not considered here). Note that Eq. (17) also involves the steady-state value of the mean-square velocity or mean kinetic energy of the particle, so unless the latter is known on other grounds, Eq. (17) does not determine the dissipation from the fluctuations, or vice versa. In thermal equilibrium, the mean kinetic energy of the particle can be independently evaluated from the MaxwellBoltzmann velocity distribution. In the present onedimensional case, the result is $\left\langle v^{2}\right\rangle=k_{B} T / m$, where $k_{B}$ is Boltzmann's constant, $T$ is the absolute temperature, and $m$ is the particle mass. Equation (17) then becomes

$$
2 \alpha k_{B} T=m\left\langle\Delta v^{2}\right\rangle .
$$

Similar relations are a typical feature of most fluctuationdissipation theorems, but they are not really essential, and the other concepts involved are equally applicable to nonequilibrium steady states in other contexts, such as turbulence.

It is straightforward to establish the connection between Eq. (18) and the usual fluctuation-dissipation theorem for the one-dimensional version of the stochastic differential Langevin equation (1), in which $\mathbf{v}$ and $\mathbf{F}$ reduce to scalars $v$ and $F$, respectively. To proceed, we must relate the discrete friction coefficient $\alpha$ to the differential friction coefficient $\beta$, and the mean-square velocity increment $\left\langle\Delta v^{2}\right\rangle$ to the amplitude of the random force $F(t)$. Both of those relations involve the mean time interval $\Delta t$ between impulses, which is no longer irrelevant and must be taken to be very short compared to the frictional time scale $m / \beta$. According to Eq. (1), the frictional change in $v$ over a time interval $\Delta t$ is $-(\beta \Delta t / m) v$. The corresponding change in the discrete Eq. (14) is $-\alpha v$, which immediately implies the identification

$$
\alpha=\frac{\beta \Delta t}{m} .
$$

The relation between $\left\langle\Delta v^{2}\right\rangle$ and $F(t)$ is a little more involved. The form of $F(t)$ required to produce a particular sequence of discontinuous velocity jumps $\Delta v_{n}$ is given by

$$
F(t)=m \sum_{n} \Delta v_{n} \delta\left(t-t_{n}\right),
$$

where $\delta(t)$ is the Dirac delta function. It follows that

$$
\begin{aligned}
\left\langle F(t) F\left(t^{\prime}\right)\right\rangle & =m^{2} \sum_{n} \sum_{k}\left\langle\Delta v_{n} \Delta v_{k}\right\rangle \delta\left(t-t_{n}\right) \delta\left(t^{\prime}-t_{k}\right) \\
& =m^{2}\left\langle\Delta v^{2}\right\rangle \delta\left(t-t^{\prime}\right) \rho(t),
\end{aligned}
$$

where

$$
\rho(t) \equiv \sum_{n} \delta\left(t-t_{n}\right),
$$

and use has been made of Eq. (12) and the properties of the delta function. Notice that the integral of $\rho(t)$ over any finite time interval is the number of pulses occurring during that interval. Thus $\rho(t)$ is the instantaneous number density of pulses per unit time. If the pulses occur randomly separated by a mean time interval $\Delta t$, then the mean number of pulses per unit time is $\overline{\rho(t)}=1 / \Delta t$. A further statistical average of Eq. (21) over the random pulse arrival times yields

$$
\overline{\left\langle F(t) F\left(t^{\prime}\right)\right\rangle}=2 B \delta\left(t-t^{\prime}\right),
$$

where

$$
B=\frac{m^{2}\left\langle\Delta v^{2}\right\rangle}{2 \Delta t} .
$$

Equations (19) and (24) combine with Eq. (18) to yield $B$ $=\beta k_{B} T$, which is the usual fluctuation-dissipation theorem for the one-dimensional Langevin equation. ${ }^{3}$ Equation (24) shows that in order for the discrete model to properly reduce to the differential Langevin equation as $\Delta t \rightarrow 0$, we must require $\left\langle\Delta v^{2}\right\rangle$ to approach zero in such a way that $\left\langle\Delta v^{2}\right\rangle / \Delta t$ remains finite in that limit, which again is suggestive of a random walk or diffusional process.

\section{CONCLUDING REMARKS}

We have discussed a simple physically transparent discrete impulsive model that exhibits the essential features of random heating and Brownian motion. It is remarkable how much interesting physics is captured by such a mathematically simple model. The model provides an elementary introduction to a number of important physical concepts that are ordinarily considered more advanced. It is hoped that this discussion will provide an accessible avenue for undergraduate and beginning graduate students in physics and engineering to acquire some valuable physical insight into those concepts prior to embarking on a study of more formal advanced treatments.

\section{ACKNOWLEDGMENTS}

The author is grateful to Katja Lindenberg and the anonymous reviewers for helpful comments and suggestions. 


\section{APPENDIX: RANDOM VARIABLES AND CORRELATIONS}

Random variables take on different values with certain probabilities. For simplicity, we restrict our attention to random variables that only take on discrete values, which can be labeled by integer subscripts. The probability that the random variable $X$ takes on the allowed value $X_{i}$ is denoted by $p\left(X_{i}\right)$. The variable $X$ is certain to take on one of its allowed values, so $\sum_{i} p\left(X_{i}\right)=1$. The average value of any function $f(X)$ that depends on $X$ is given by

$$
\langle f(X)\rangle=\sum_{i} p\left(X_{i}\right) f\left(X_{i}\right)
$$

Many situations involve more than one random variable. The probability that two random variables $X$ and $Y$ simultaneously take on the values $X_{i}$ and $Y_{j}$ is called their joint probability, and is denoted by $p\left(X_{i}, Y_{j}\right)$. The average of a function $f(X, Y)$ that depends on both $X$ and $Y$ is given by

$$
\langle f(X, Y)\rangle=\sum_{i j} p\left(X_{i}, Y_{j}\right) f\left(X_{i}, Y_{j}\right) .
$$

The probability that $X$ takes on the value $X_{i}$ regardless of the value of $Y$ is the sum of $p\left(X_{i}, Y_{j}\right)$ over all possible values of $Y_{j}$, so that

$$
p\left(X_{i}\right)=\sum_{j} p\left(X_{i}, Y_{j}\right)
$$

The probability that $X$ takes on the value $X_{i}$ when $Y$ is known to have the value $Y_{j}$ is the conditional probability of $X$ given $Y$, and is denoted by $p\left(X_{i} \mid Y_{j}\right)$. A little thought shows that

$$
p\left(X_{i}, Y_{j}\right)=p\left(X_{i} \mid Y_{j}\right) p\left(Y_{j}\right)
$$

The random variables $X$ and $Y$ are said to be statistically independent when the probability that $X$ will take on the value $X_{i}$ is independent of $Y$; that is, when $p\left(X_{i} \mid Y_{j}\right)$ does not depend on $Y_{j}$. In this case, summing Eq. (A4) over $j$ shows that $p\left(X_{i} \mid Y_{j}\right)=p\left(X_{i}\right)$, and Eq. (A4) reduces to

$$
p\left(X_{i}, Y_{j}\right)=p\left(X_{i}\right) p\left(Y_{j}\right)
$$

When $X$ and $Y$ are statistically independent so that Eq. (A5) is satisfied, averages of products of functions of $X$ and $Y$ alone factor into products of their averages,

$$
\begin{aligned}
\langle f(X) g(Y)\rangle & =\sum_{i j} p\left(X_{i}, Y_{j}\right) f\left(X_{i}\right) g\left(Y_{j}\right) \\
& =\sum_{i j} p\left(X_{i}\right) p\left(Y_{j}\right) f\left(X_{i}\right) g\left(Y_{j}\right) \\
& =\left[\sum_{i} p\left(X_{i}\right) f\left(X_{i}\right)\right]\left[\sum_{j} p\left(Y_{j}\right) g\left(Y_{j}\right)\right] \\
& =\langle f(X)\rangle\langle g(Y)\rangle .
\end{aligned}
$$

The most common and important special case of this relation is

$$
\langle X Y\rangle=\langle X\rangle\langle Y\rangle \text {. }
$$

Random variables that satisfy Eq. (A7) are said to be uncorrelated. Note that the converse is not necessarily true; it is possible for $X$ and $Y$ to be uncorrelated in the sense of Eq. (A7) without being statistically independent.

The preceding relations easily generalize to the case in which the random variables are continuous. The main features of this generalization are that the discrete quantities $\left(X_{i}, Y_{j}, \ldots\right)$ are replaced by corresponding continuous variables $(x, y, \ldots)$, probabilities like $p\left(X_{i}\right)$ are replaced by corresponding probability densities or distribution functions $p_{X}(x)$ (defined such that $p_{X}(x) d x$ is the probability that $x$ $\leq X \leq x+d x)$, and sums over $(i, j, \ldots)$ are replaced by integrals over the corresponding continuous variables $(x, y, \ldots)$.

${ }^{1}$ D. A. McQuarrie, Statistical Mechanics (Harper \& Row, New York, 1976).

${ }^{2}$ N. G. van Kampen, Stochastic Processes in Physics and Chemistry, 2nd ed. (North-Holland, Amsterdam, 1992).

${ }^{3}$ R. Zwanzig, Nonequilibrium Statistical Mechanics (Oxford U. P., New York, 2001).

${ }^{4}$ B. J. West and K. Lindenberg, "Nonlinear fluctuation-dissipation relations," in Fluctuations and Sensitivity in Nonequilibrium Systems, edited by W. Horsthemke and D. K. Kondepudi (Springer-Verlag, Berlin, 1984), p. 233.

${ }^{5}$ J. D. Ramshaw and K. Lindenberg, "Augmented Langevin description of multiplicative noise and nonlinear dissipation in Hamiltonian systems," J. Stat. Phys. 45, 295-307 (1986).

${ }^{6} \mathrm{~K}$. Lindenberg and B. J. West, The Nonequilibrium Statistical Mechanics of Open and Closed Systems (VCH, New York, 1990).

${ }^{7}$ S. Chapman and T. G. Cowling, The Mathematical Theory of NonUniform Gases, 3rd ed. (Cambridge U. P., Cambridge, 1970).

${ }^{8}$ R. E. London, "Force-velocity cross correlations and the Langevin equation," J. Chem. Phys. 66, 471-479 (1977).

${ }^{9}$ S. Chandrasekhar, "Stochastic problems in physics and astronomy," Rev. Mod. Phys. 15, 1-89 (1943).

${ }^{10}$ M. N. Barber and B. W. Ninham, Random and Restricted Walks: Theory and Applications (Gordon and Breach, New York, 1970).

${ }^{11}$ G. H. Weiss and R. J. Rubin, "Random walks: Theory and selected applications," Adv. Chem. Phys. 52, 363-505 (1983).

${ }^{12}$ Random Walks and Their Applications in the Physical and Biological Sciences, edited by M. F. Shlesinger and B. J. West, AIP Conference Proceedings, No. 109 (American Institute of Physics, New York, 1984). 